\title{
Echocardiographic Abnormalities in New-onset Polymyositis/dermatomyositis
}

\author{
Andrea Péter, Ágnes Balogh, Szabolcs Szilágyi, Réka Faludi, Melinda Nagy-Vincze, \\ István Édes, and Katalin Dankó
}

ABSTRACT. Objective. To identify early echocardiographic abnormalities at the time of diagnosis of polymyositis (PM) and dermatomyositis (DM) and follow the echocardiographic findings during the first 3 months of therapy.

Methods. We included 30 PM/DM patients (23/7) with a mean age of $42.3 \pm 1.6$ years and without cardiovascular symptoms. Age-matched healthy patients served as controls. Clinical characteristics were recorded. Traditional echocardiography and tissue Doppler imaging (TDI) were performed to measure systolic [ejection fraction, right ventricular fractional area change (RV FAC), lateral and tricuspid annulus s velocities] and diastolic echocardiographic variables (mitral inflow velocities: E, A; deceleration time: DT; lateral and tricuspid annulus e', a' velocities, lateral E/e').

Results. The left and right ventricular systolic dysfunction detected by TDI at the time of the $\mathrm{PM} / \mathrm{DM}$ diagnosis improved, and characteristic values at the end of the followup period were comparable to those of the controls (lateral s: $10.6 \pm 0.2,8.7 \pm 0.4,9.6 \pm 0.3,11.3 \pm 0.2 \mathrm{~cm} / \mathrm{s}$; RV FAC: $45.2 \pm 2.3,36.9 \pm 1.5,42.2 \pm 1.3,46.9 \pm 1.2 \%$; tricuspid s: $13.3 \pm 0.2,9.5 \pm 0.4,10.3 \pm 0.3$, $11.6 \pm 0.5 \mathrm{~cm} / \mathrm{s}$; control, 0,1 , and $3 \mathrm{mos}$, respectively). Measurements indicated the development of diastolic dysfunction at 3 mos (E/A: $1.4 \pm 0.1,1.29 \pm 0.05,1.03 \pm 0.05,0.92 \pm 0.05$; DT: $148.6 \pm$ $3.6,157.3 \pm 5.7,168.3 \pm 6.0,184.3 \pm 6.2 \mathrm{~ms}$; lateral e': $12.8 \pm 0.3,12.1 \pm 0.5,10.2 \pm 0.6,10.8 \pm 0.8$ $\mathrm{cm} / \mathrm{s} ; \mathrm{E} / \mathrm{e}^{\prime}: 5.6 \pm 0.1,5.0 \pm 0.22,6.92 \pm 0.46,7.64 \pm 0.47$; control, 0,1 , and 3 mos, respectively).

Conclusion. TDI is a useful method to detect early cardiac abnormalities complementing the conventional echocardiographic measurements. LV and RV systolic dysfunction found in the acute phase significantly improved during the first 3 months of therapy; however, deterioration of diastolic dysfunction was also observed. (First Release Dec 1 2014; J Rheumatol 2015;42:272-81; doi:10.3899/jrheum.140626)

\author{
Key Indexing Terms: \\ POLYMYOSITIS \\ TISSUE DOPPLER IMAGING
}

\section{DERMATOMYOSITIS ECHOCARDIOGRAPHY} SYSTOLIC AND DIASTOLIC DYSFUNCTION
Idiopathic inflammatory myopathies are rare immune-mediated chronic inflammatory diseases characterized by symmetric and progressive weakness of the proximal muscles. Among them, polymyositis (PM) and dermatomyositis (DM) are characterized by chronic inflammation, fibrosis, and damage and destruction of the muscle fibers ${ }^{1}$. The pathomechanism in PM involves perivascular infiltration caused by CD8+ cytotoxic T cells and macrophages, while inflammation and skin rash in DM is mediated

From the Institute of Cardiology, and the Institute of Internal Medicine, University of Debrecen, Debrecen; Heart Institute, University of Pécs, Pécs, Hungary; Cardiothoracic Centre, Freeman Hospital, Newcastle upon Tyne, UK.

A. Péter, MD; Á. Balogh, MD, PhD, Institute of Cardiology, University of Debrecen; S. Szilágyi, MD, PhD, Cardiothoracic Centre, Freeman Hospital; R. Faludi, MD, PhD, Heart Institute, University of Pécs; M. Nagy-Vincze, $M D$, Institute of Internal Medicine, University of Debrecen; I. Édes, $M D, P h D, D S c$, Institute of Cardiology, University of Debrecen; K. Dankó, MD, PhD, DSc, Institute of Internal Medicine, University of Debrecen.

Address correspondence to Dr. A. Péter, Institute of Cardiology, University of Debrecen, Móricz Zs. krt. 22, H-4032 Debrecen, Hungary. E-mail:doktorpandi@gmail.com

Accepted for publication October 9, 2014. predominantly by B cell-dependent humoral mechanisms and Th cell-mediated cytokines.

Cardiac involvement in DM was first published in 1899 by Oppenheim ${ }^{2}$. Numerous studies have since confirmed that cardiac involvement is common and is one of the main prognostic factors in $\mathrm{PM} / \mathrm{DM}^{3,4,5,6}$ because extramuscular and cardiac manifestations are associated with worse prognosis. Pathologic findings of the myocardium including active myocarditis, mononuclear infiltration, focal fibrosis, vasculitis, intima proliferation, and media sclerosis of the vessels indicate that the heart is a target organ of $\mathrm{PM} / \mathrm{DM}^{7}$. A metaanalysis (1975-2011; 26 publications, 1530 patients) concluded that the incidence of cardiac involvement in myositis varies between $9 \%$ and $72 \%$. The most common clinical syndrome was heart failure in $32 \%-77 \%$ of the patients. Left ventricular (LV) diastolic dysfunction was found in $42 \%$ of the patients, while conduction disorders occurred in $25 \%-38.5 \%$ and hyperkinetic LV contraction was seen in $6 \%-12 \%$. Finally, the cause of death was identified as the direct consequence of heart disease in $46.3 \%$ of the patients ${ }^{4}$. Studies suggest that subclinical

Personal non-commercial use only. The Journal of Rheumatology Copyright (C) 2015. All rights reserved. 
cardiac manifestations are more common ${ }^{8}$, but details are yet to be clarified. The incidence depends on the applied diagnostic method, patient selection, disease setting, study type, and whether subclinical or clinical manifestations are allowed. The diagnosis of autoimmune myocardial involvement is difficult; routine echocardiography (ECG) and laboratory tests complemented with echocardiography, cardiac magnetic resonance imaging (MRI), and the rarely used endomyocardial biopsy can further improve the diagnostic probability.

Few prospective, detailed echocardiographic studies involving large numbers of patients are available in the literature about cardiovascular involvement in this group of patients. These results mainly included early LV diastolic dysfunction $9,10,11$, which may serve as a precursor for systolic and diastolic heart failure. Lu and coworkers found in patients with PM/DM that female sex, late onset, and long disease course were 3 independent risk factors in the development of LV diastolic dysfunction ${ }^{9}$. Moreover, Wang, et al demonstrated a significant relationship between the changes in the diastolic variables and the disease duration in patients with DM, similar to what has been shown in rheumatoid arthritis (RA) and systemic sclerosis ${ }^{11}$.

In our study, conventional echocardiographic measurements were completed with data obtained by the tissue Doppler imaging (TDI) method. This approach is sensitive enough for the recognition of both structural and functional subclinical alterations of the myocardium. It ensures better description of the LV and right ventricular (RV) systolic function as well as the LV filling pressure and diastolic function ${ }^{12,13}$. We characterized the systolic and diastolic function of the LV and RV functional variables in patients with $\mathrm{PM} / \mathrm{DM}$ at the time of the diagnosis. Additionally, these variables were followed during the first 3 months of the therapy.

\section{MATERIALS AND METHODS}

Study population. A total of 30 hospitalized PM/DM patients with recent onset [(23/7), female/male (27/3)] and without clinical cardiac symptoms were enrolled from the Department of Internal Medicine (Debrecen, Hungary) between January 2012 and January 2013, with a mean age of 42.3 \pm 1.6 years. All patients fulfilled the criteria of either definitive PM or DM as proposed by Bohan and Peter ${ }^{14}$. Patients were excluded if they presented with malignancy, systemic autoimmune diseases (RA, systemic lupus erythematosus, mixed connective tissue disease), overlap syndromes, previously diagnosed congenital heart disease, rheumatic fever, hypertension (HTN), coronary artery disease, valvular heart disease, cardiomyopathy, arrhythmias, diabetes mellitus, severe renal disease (serum creatinine level $\geq 130 \mu \mathrm{mol} / \mathrm{l}$ ), anemia (hemoglobin $\leq 13.5 \mathrm{~g} / \mathrm{dl}$ in male, $\leq 12.0 \mathrm{~g} / \mathrm{dl}$ in female), atrial fibrillation, or mitral regurgitation exceeding moderate severity.

The patients were thoroughly examined at the time of diagnosis $(0$ month), when anamnestic data were collected, physical examination involving blood pressure measurement and ECG was performed, and blood samples were collected for routine and special autoimmune laboratory tests. Finally, echocardiographic scan was performed, obtaining a dataset with conventional and TDI measurements. Thereafter, specific therapy began and the same echocardiographic examinations were repeated 1 month and 3 months after the diagnosis. An age-matched healthy population served as control (mean age $44.4 \pm 0.9 \mathrm{yrs}$ ). The study protocol was approved by the Ethics Committee of the University of Debrecen, and written informed consent was obtained from each participant. The study was conducted according to the Declaration of Helsinki.

Clinical characteristics. Detailed records of clinical information were made at onset, including age, blood pressure, heart rate, muscle disease activity, presence of lung involvement, and Raynaud sign. Muscle disease activity was evaluated according to the Myositis Disease Activity Assessment Tool (MDAAT, version 2, 2005) ${ }^{15}$ by physician assessment using a visual analog score (VAS). High-resolution computed tomography of the lungs was performed to investigate radiographic abnormalities [pulmonary infections, fibrosis, tuberculosis, and interstitial lung disease (ILD)]. Laboratory assessment performed on the first day of hospitalization included, among other items, total cholesterol, triglyceride, blood glucose, uric acid, total creatine kinase (CK), and an autoimmune panel (anti-dsDNA, anti-SRP, anti-Scl-70, and myositis profile 3 Blot Strip: Ro52, OJ, EJ, Pl-12, Pl-7, SRP, anti-Jo1, PM-Scl75, PM-Sc1100, Ku, and Mi-2B). After the diagnosis was established, specific therapy involved high-dose (0.5-1-2-3 mg/kg/day) corticosteroid (prednisolone or methylprednisolone) for 4 weeks, which was tapered gradually (in 10 weeks, decreased by $4 \mathrm{mg} /$ week). We used proton pump inhibitors, potassium, and diuretic (furosemide) to prevent the side effects of corticosteroids (gastrointestinal ulcers, bleeding, hypokalemia, and fluid retention) as supplementary therapy. Beta blockers were also needed in $30 \%$ of the cases. Second-line treatment was introduced when a patient's general condition deteriorated despite having taken corticosteroids or when severe dysphagia or lung fibrosis occurred ${ }^{1}$. Cyclosporine $(5 \mathrm{mg} / \mathrm{kg} /$ day, reduced for $2.5-3.5$ $\mathrm{mg} / \mathrm{kg} / \mathrm{day}$ after 1-2 mos) was added to the corticosteroid therapy in patients with ILD and no improvement while taking steroids.

Conventional echocardiography. Transthoracic echocardiography was performed using ultrasound equipment (Acuson Sequoia, Siemens AG) with a $1-5 \mathrm{MHz}$ transducer. All measurements were carried out by a single observer blinded to patient/control status. All patients were examined in the standard left parasternal and apical windows with normal breathing. Left atrial (LA) diameter, LV end-diastolic and end-systolic diameters were determined with 2D and $\mathrm{M}$ mode echocardiography based on the criteria of the European Association of Echocardiography ${ }^{16}$. LV ejection fraction (EF) was calculated according to the Simpson method ${ }^{17}$. Mitral inflow velocities were evaluated by pulsed-wave Doppler imaging on the apical 4-chamber view with the sample volume placed at the tip of the mitral leaflets. Peak early (E) and peak late diastolic transmitral flow velocities (A) were measured and E/A ratio was calculated to characterize LV diastolic function. Dependence of the transmitral inflow on preload, heart rate, and contractility is the main limitation for this method. Moreover, the early transmitral flow deceleration time (DT), a further marker of LV diastolic function, was also measured. RV function was described by the fractional area change [RV FAC: (end-diastolic RV area - end-systolic RV area)/end-diastolic RV area]. Mitral annulus plane systolic excursion (MAPSE) and tricuspid annulus plane systolic excursion (TAPSE) were measured by M mode to determine the longitudinal function of the left and right ventricle, respectively. TAPSE was calculated by placing the M mode cursor through the tricuspid annulus in a standard apical 4-chamber window and the longitudinal excursion of the endocardial surface between end-diastole and end-systole was measured. MAPSE was obtained by similar method in the mitral annulus. Pulmonary artery systolic pressure (PASP) was calculated with the measurement of the tricuspid regurgitation peak velocity in those patients who had some degree of tricuspid regurgitation. In the absence of obstruction to RV outflow tract, RV systolic pressure equals PASP. Mean values of measurements from 3 consecutive beats were calculated for further analysis.

TDI measurements. All TDI measurements were performed in adherence to the guidelines and standards of the European Society of Echocardiography.

Personal non-commercial use only. The Journal of Rheumatology Copyright @ $\subset$ 2015. All rights reserved 
TDI uses Doppler principles to measure the velocity of myocardial motions: peak myocardial systolic velocity (s), early myocardial diastolic velocity ( $\left.e^{\prime}\right)$ and late myocardial diastolic velocity $\left(a^{\prime}\right)$. Pulsed wave tissue Doppler was performed in both groups with a frame rate of about 60/s. TDI data were obtained from the lateral site of the mitral and tricuspid annulus. The s velocity at the lateral annulus is a good measure of the longitudinal systolic funcion ${ }^{18}$. In the absence of regional wall-motion abnormalities, TDI velocities obtained from the posterior and septal mitral annuli do not provide further information about the ventricular function, therefore only the velocities of the lateral sites were recorded. The e' velocity of the mitral and tricuspid annulus is an established indicator of LV and RV myocardial relaxation, respectively. When myocardial relaxation is impaired because of aging or a disease process, $\mathrm{e}^{\prime}$ is less or not at all affected by the preload. The a' velocity of the mitral annulus has been correlated with LA function, which is increased in early diastolic dysfunction, and decreases when atrial function deteriorates ${ }^{19}$. Moreover, E/e' ratio, a preload-independent factor, can be applied for the estimation of the $\mathrm{LV}$ filling pressure. $\mathrm{E} / \mathrm{e}^{\prime} \leq 8$ indicates normal LV end-diastolic pressure ${ }^{20}$.

Statistical analysis. Numerical data in this report are given as mean values \pm SEM. D'Agostino-Pearson normality test was used to test whether the data showed a Gaussian distribution. The Kruskal-Wallis test or ANOVA followed by the Bonferroni posthoc test were used to compare the different groups, with $\mathrm{p}$ values $<0.05$ considered statistically significant. Statistical analyses were performed with GraphPad Prism 5.02 software (GraphPad Software Inc.).

\section{RESULTS}

Baseline characteristics. Table 1 demonstrates the general characteristics of the patients. Of the 30 patients, 23 had PM and 7 had DM. The study population was chosen randomly and matched the general prevalence findings in our country. The patients did not have HTN. Physiologic mean blood pressure was recorded (systolic RR: $120 \pm 2 \mathrm{mmHg}$, diastolic RR: $76 \pm 1 \mathrm{mmHg}$ ), and the mean heart rate was 85 \pm 1 beats/min. Neither specific findings during physical examination nor ECG abnormalities were found. The autoantibody profile is detailed also in Table 1 .

Echocardiographic findings. At the time of diagnosis, chamber diameters - similarly to those in the control group - were in the normal range and remained unchanged during the followup period (Table 2). Echocardiographic measurements were done to evaluate the systolic and diastolic function of the LV and RV.

$L V$ systolic function. Global systolic function of the LV was characterized by the traditionally used variable, the EF measured by the Simpson method, which was in the normal range, suggesting normokinetic LV throughout the 3 months (Figure 1, Table 2). The longitudinal LV systolic function (MAPSE) assessed by M mode increased significantly for the third month compared to the baseline value (Figure 1, Table 2). Additionally, LV systolic function measured by the more sensitive and detailed TDI method showed major changes: the mean mitral lateral systolic velocity (lateral s) was significantly lower than that of the controls; it increased to the control level in the following 3 months (Figures 1 and 4 , Table 2). These traditionally measured data suggest a physiological global systolic LV function; nevertheless, an initial deteriorated LV systolic function was observed by the
Table 1. Clinical characteristics and autoantibody profile of the patients with polymyositis/dermatomyositis.

\begin{tabular}{|c|c|}
\hline Patient Characteristics & Frequency/mean \\
\hline \multicolumn{2}{|l|}{ Diagnosis } \\
\hline Polymyositis & $77 \%$ \\
\hline Dermatomyositis & $23 \%$ \\
\hline \multicolumn{2}{|l|}{ Sex } \\
\hline Male & $10 \%$ \\
\hline Female & $90 \%$ \\
\hline Mean age at onset, yrs, \pm SD & $42.3 \pm 1.6$ \\
\hline Raynaud sign & $33.3 \%$ \\
\hline Proximal muscle weakness & $87 \%$ \\
\hline Neck muscle weakness & $3.3 \%$ \\
\hline Distal muscle weakness & $3.3 \%$ \\
\hline Myalgia/arthralgia & $10 \%$ \\
\hline Weight loss & $3.3 \%$ \\
\hline Dysphagia & $16.7 \%$ \\
\hline Erythema & $16.7 \%$ \\
\hline Lung disease & $36.7 \%$ \\
\hline $\mathrm{SBP}, \mathrm{mmHg}$ & $120 \pm 2$ \\
\hline $\mathrm{DBP}, \mathrm{mmHg}$ & $76.1 \pm 1$ \\
\hline $\mathrm{HR}$, beat $/ \mathrm{min}$ & $85 \pm 1$ \\
\hline Serum cholesterol, mmol/1 & $4.65 \pm 0.13$ \\
\hline Serum triglyceride, $\mathrm{mmol} / \mathrm{l}$ & $1.41 \pm 0.11$ \\
\hline Blood glucose, mmol/1 & $5.08 \pm 0.18$ \\
\hline Uric acid, $\mu \mathrm{mol} / 1$ & $289.4 \pm 12.51$ \\
\hline CK activity, U/1 & $1065.23 \pm 222.38$ \\
\hline \multicolumn{2}{|l|}{ Muscle histology } \\
\hline Negative & $13.3 \%$ \\
\hline Mononuclear infiltration & $80 \%$ \\
\hline Chronic inflammation & $6.7 \%$ \\
\hline \multicolumn{2}{|l|}{ Specific therapy } \\
\hline Steroid & $100 \%$ \\
\hline Cyclosporine & $13.3 \%$ \\
\hline Anti-dsDNA & $0 \%$ \\
\hline Anti-EJ (glycyl-tRNA synthetase) & $0 \%$ \\
\hline Anti-Jo1 (histidyl-tRNA synthetase) & $20 \%$ \\
\hline Anti-Ku (Ku antigen) & $0 \%$ \\
\hline Anti-Mi-2B (nuclear helicase) & $0 \%$ \\
\hline Anti-OJ (isoleucyl-tRNA synthetase) & $0 \%$ \\
\hline Anti-PL-12 (alanyl-tRNA synthetase) & $0 \%$ \\
\hline Anti-PL-7 (threonyl-tRNA synthetase) & $0 \%$ \\
\hline Anti-PM/Scl-100 (PM/Scl-100 antigen) & $3.3 \%$ \\
\hline Anti-PM/Scl-75 (PM/Scl-75 antigen) & $10 \%$ \\
\hline Anti-Ro-52 (Ro-52 antigen) & $20 \%$ \\
\hline Anti-Scl-70 (type I topoisomerase) & $43.3 \%$ \\
\hline Anti-SRP (signal recognition particle) & $0 \%$ \\
\hline
\end{tabular}

Autoantibodies' targets are listed in parentheses. SBP: systolic blood pressure; DBP: diastolic blood pressure; HR: heart rate; CK: creatine kinase.

TDI method. The function improved parallel with the administration of the immunosuppressive therapy.

$L V$ diastolic function. LV diastolic function was evaluated by the characterization of the transmitral inflow Doppler pattern (E/A ratio, DT) and the TDI measurement of the lateral segment of the LV myocardium (lateral $\mathrm{e}^{\prime}$ and $\mathrm{a}^{\prime}$ velocities). E/A ratio calculated from the Doppler trace of the transmitral inflow fit the normal range at the time of the diagnosis with significant decrease thereafter (Figures 2 and 
Table 2. Echocardiographic variables measured in the control group and in patients with polymyositis/dermatomyositis.

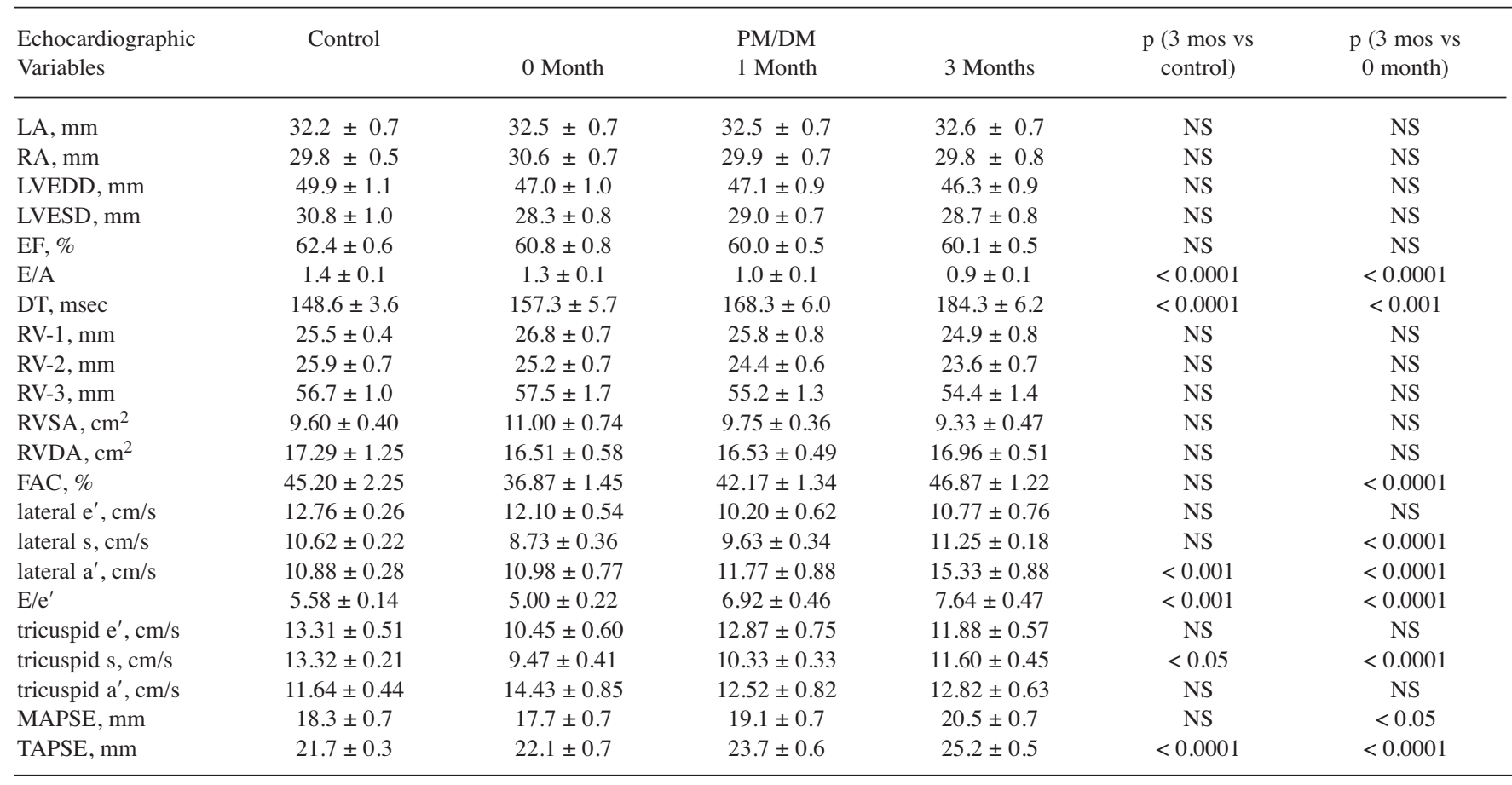

PM: polymyositis; DM: dermatomyositis; LA: left atrial diameter; RA: right atrial diameter; LVEDD: left ventricular end-diastolic diameter; LVESD: left ventricular end-systolic diameter; EF: ejection fraction; E: peak early diastolic velocity; A: peak late diastolic velocity; DT: deceleration time; RV: right ventricular diameter measured at 3 different levels; RVSA: right ventricular systolic area; RVDA: right ventricular diastolic area; FAC: fractional area change; lateral e': early myocardial diastolic velocity; lateral s: peak systolic mitral annulus velocity; lateral a': late myocardial diastolic velocity; tricuspid e': early tricuspid annular diastolic velocity; tricuspid s: peak systolic tricuspid annulus velocity; tricuspid a': late tricuspid annular diastolic velocity; MAPSE: mitral annulus plane systolic excursion; TAPSE: tricuspid annulus plane systolic excursion.

a

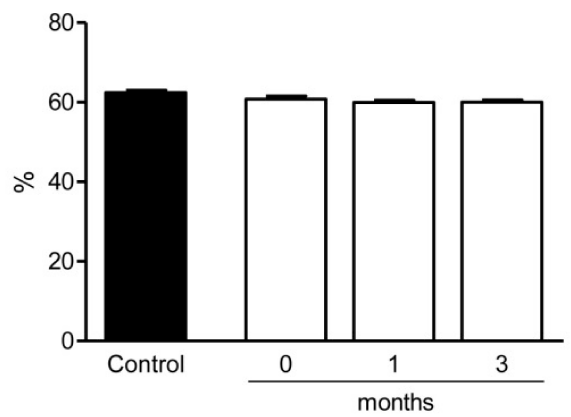

b

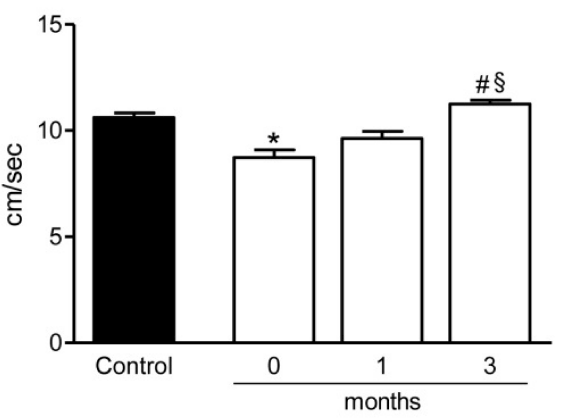

$\mathrm{EF}$

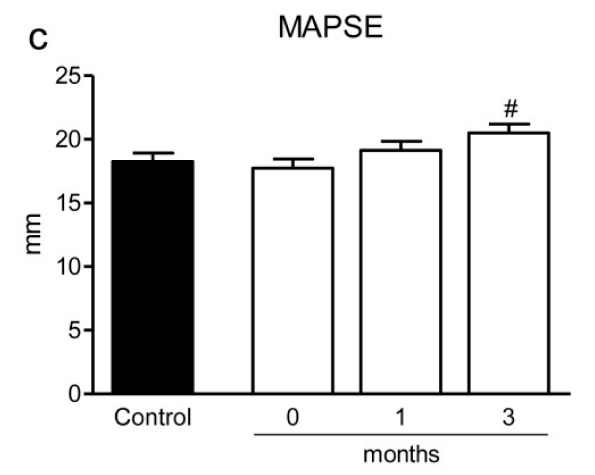

Figure 1. Followup of the echocardiographic variables characterizing left ventricular systolic function. Ejection fraction (EF) measured by the Simpson method suggested normokinetic left ventricular systolic function during the whole followup period (a). In contrast, lateral systolic velocity (lateral s) measured by tissue Doppler imaging was lower compared to controls at the time of diagnosis and it normalized by the end of the third month (b). In parallel, the mitral annulus plane systolic excursion (MAPSE) increased within the normal range significantly in the third month (c). ${ }^{*} \mathrm{p}<0.05$ vs control; \# $\mathrm{p}<0.05$ vs 0 month, $\S \mathrm{p}<0.05$ vs 1 month. 
a

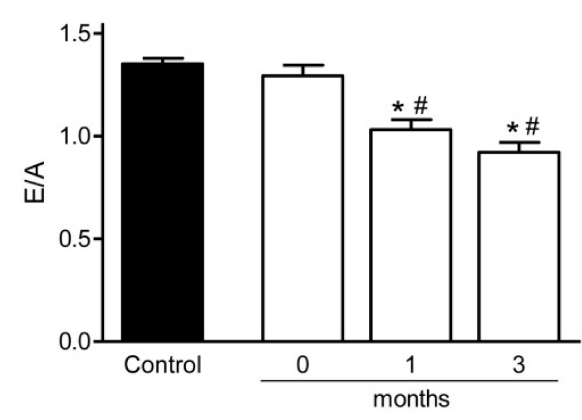

C

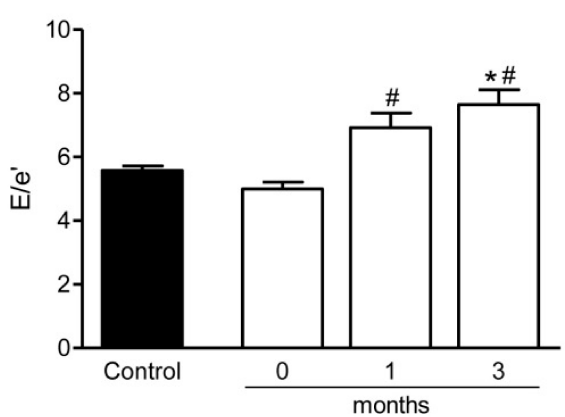

b

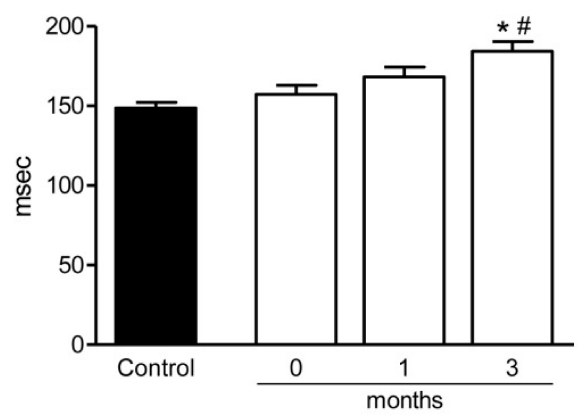

d

lateral $e^{\prime}$

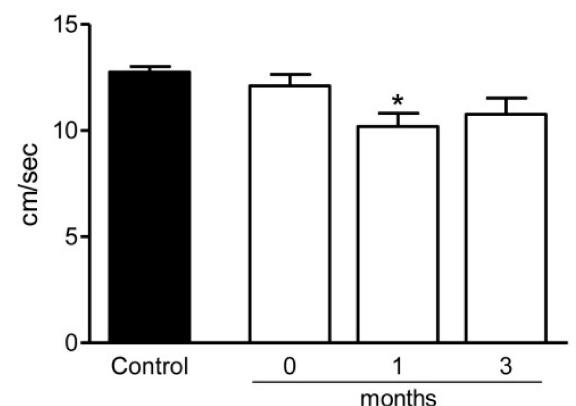

Figure 2. Left ventricular diastolic dysfunction developed 3 months after the diagnosis. E/A ratio (E: peak early diastolic velocity, A: peak late diastolic velocity) calculated from the transmitral flow was in the normal range (E/A $>1$ ) at time of diagnosis, but it decreased significantly, revealing diastolic dysfunction in the followup period (a). Deceleration time (DT) increased significantly (b), while the early diastolic myocardial relaxation velocity (lateral $\mathrm{e}^{\prime}$ ) showed a decreasing tendency (d). The increasing E/e' ratio during the followup period also supported the development of the diastolic dysfunction (c). ${ }^{*} \mathrm{p}<0.05$ vs control, \# p $<0.05$ vs 0 month.

4, Table 2). In line with this variable, DT increased significantly (Figure 2, Table 2) during the followup period. In parallel, the early diastolic lateral myocardial relaxation velocity (lateral $\mathrm{e}^{\prime}$ ) decreased significantly for the first month of the followup period, and showed a decreasing tendency compared to the control in the third month (Figures 2 and 4, Table 2). The late diastolic lateral myocardial relaxation velocity (lateral a') was significantly higher in the third month compared both to the healthy controls and to the baseline value of the patients with $\mathrm{PM} / \mathrm{DM}$ (Figure 4, Table 2). E/e' ratio - calculated from the mitral inflow E velocity and the TDI lateral $\mathrm{e}^{\prime}$ velocity - is commonly used to estimate the LV filling pressure. At the time of the diagnosis the finding in the patients with $\mathrm{PM} / \mathrm{DM}$ was similar to that in the controls. This value increased significantly during the followup period; however, it did not reach the cutoff value of 8 , which would suggest an elevated LV filling pressure (Figure 2, Table 2). These data show that diastolic dysfunction occurred during the followup period; however, no significant correlation could be established between the diastolic variables and disease activity assessed by MDAAT VAS score (E/A: $\mathrm{r}^{2}=0.7181$, $\left.\mathrm{p}=0.3563 ; \mathrm{E} / \mathrm{e}^{\prime}: \mathrm{r}^{2}=0.6961, \mathrm{p}=0.3717\right)$.
Pulmonary artery systolic pressure and RV function. Pulmonary HTN could not be found in our study population: the average PASP was $34.17 \pm 1.91 \mathrm{mmHg}$ and it did not change significantly during the 3 months $(34.71 \pm 1.61$; $34.12 \pm 1.81,1$ and 3 mos, respectively), indicating normal PASP, thereby pulmonary HTN is unlikely during the 3 -month followup period in our study population. RV systolic function characterized by the FAC significantly improved during the 3 months compared to the baseline value below the physiological range (Figure 3, Table 2). In line, the longitudinal RV systolic function (TAPSE) increased significantly for the third month compared to the initial value (Figures 3 and 4, Table 2). Moreover, the tricuspid annular systolic velocity (tricuspid s) was significantly reduced in the patients with PM/DM compared to the control group at any time, but it increased significantly during the followup period, approaching the control normokinetic RV systolic function (Figure 3, Table 2). The tricuspid early diastolic velocity (tricuspid $\mathrm{e}^{\prime}$ ) - characterizing RV diastolic function - showed opposite changes compared to the lateral $\mathrm{e}^{\prime}$ in the left heart, from a subnormal level in patients with $\mathrm{PM} / \mathrm{DM}$ at the time of the diagnosis, it increased significantly for the first month of followup. Also, tricuspid a' velocity showed a tendency to increase in the 


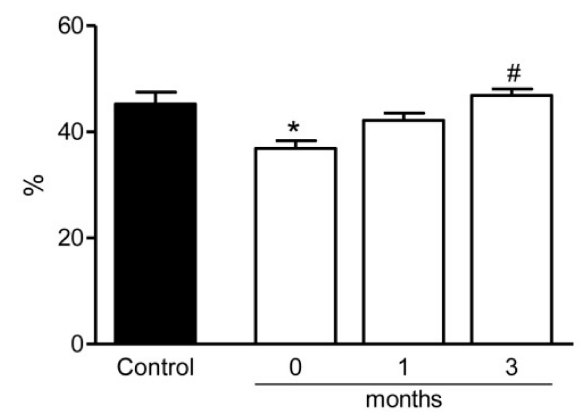

b

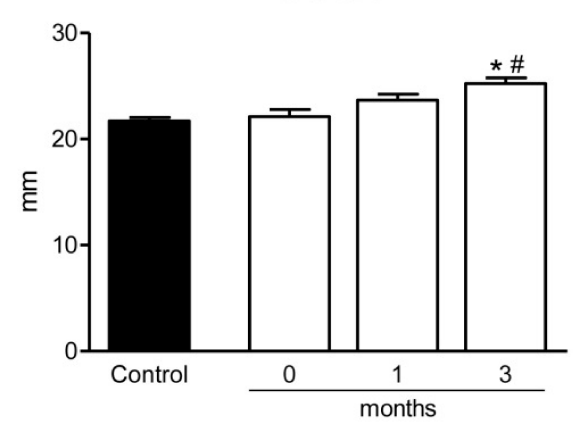

C

Figure 3. Changes in the right ventricular systolic function followed by echocardiography. Fractional area change (FAC) revealed a right ventricular systolic dysfunction at time of diagnosis that reached the normal level at the end of the followup period (a). The tricuspid annulus plane systolic excursion (TAPSE) increased significantly (b); moreover the tricuspid systolic velocity (tricuspid s) increased significantly from the lower initial level $(\mathrm{c})$. $* \mathrm{p}<$ 0.05 vs control, \# p $<0.05$ vs 0 month.

patient group, but the differences did not reach the level of significance. Besides the LV, both the systolic and diastolic function of the RV seem to be affected in patients with $\mathrm{PM} / \mathrm{DM}$

\section{DISCUSSION}

This is the first study, to our knowledge, to reveal LV and RV systolic dysfunction with the TDI method in middle-aged patients with PM/DM without cardiovascular symptoms early after diagnosis. These abnormalities improved significantly during the followup period, while having been treated with immunosuppressive therapy. In addition, specific echocardiographic data indicated diastolic dysfunction in accordance with the findings of others ${ }^{9,10,11}$. These results highlight the importance of TDI measurements in the identification of early signs of cardiac involvement in PM/DM, a critical prognostic factor in these diseases.

Conventional echocardiographic measurements showed physiological RV, LV, and atrial diameters and normokinetic LV systolic function initially. Lateral systolic velocity measured by the more sensitive TDI method, however, suggested subclinical LV systolic dysfunction. This variable returned to the normal level after a 3 -month specific therapy.
Similarly to the LV variables, RV systolic dysfunction was reflected by the decreased tricuspid systolic velocity and FAC detected at baseline. While the tricuspid systolic velocity only approached the control level after 3 months, the FAC normalized by the end of the followup period. In the initial phase of the disease, at the time of the diagnosis the TDI results revealing LV and RV systolic dysfunction may be the early signs of the cardiac involvement, e.g., acute myocarditis or myocardial edema. During the followup period characterized by high-dose steroid administration, normalization of the lateral and tricuspid systolic velocities measured by TDI may indicate attenuating myocardial edema as a response for the specific therapy. Previous autopsy data reporting myocarditis in $25 \%-30 \%$ of patients with autoimmune myositis also support this proposal $^{7,21}$.

Interestingly, although physiological LV diastolic function was found at the time of the diagnosis, diastolic dysfunction developed during the followup period, as reflected by the reduced e' velocity, the decreased E/A ratio, and the significant increase in the DT and the E/e' ratio. The increasing E/e' ratio did not exceed the upper limit of the physiological range, revealing normal LV filling pressure at this timepoint ${ }^{22}$. The above changes in the echocardio-

$$
\text { Personal non-commercial use only. The Journal of Rheumatology Copyright ( }) \text { 2015. All rights reserved. }
$$



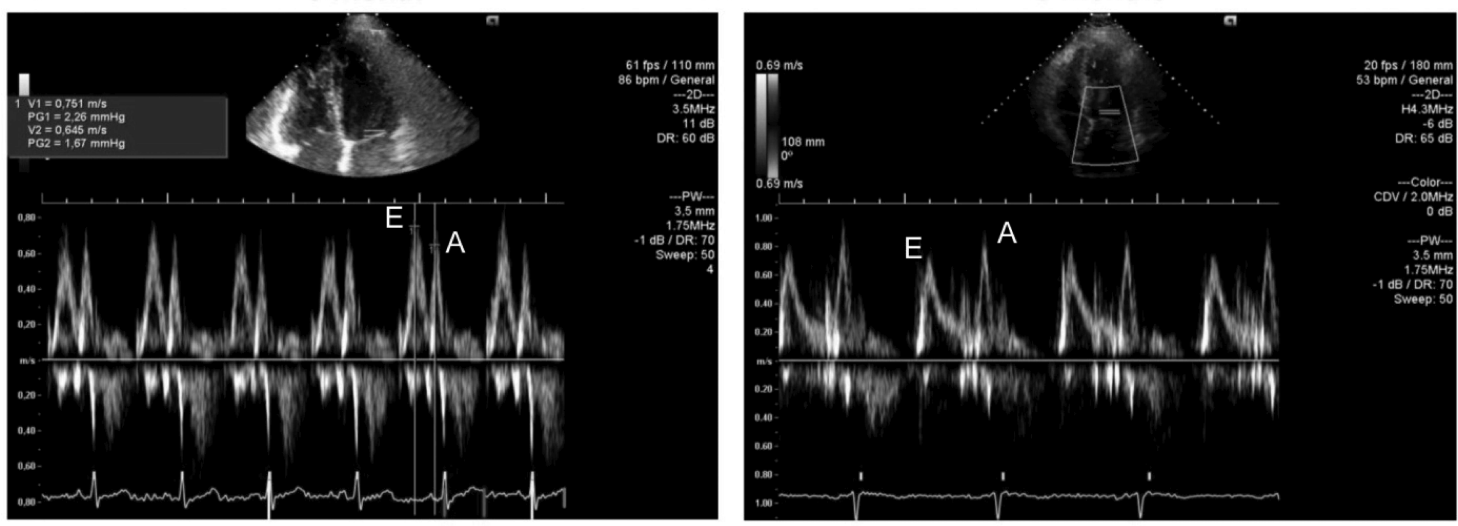

b

0 month

TDI of the lateral mitral annulus

3 months
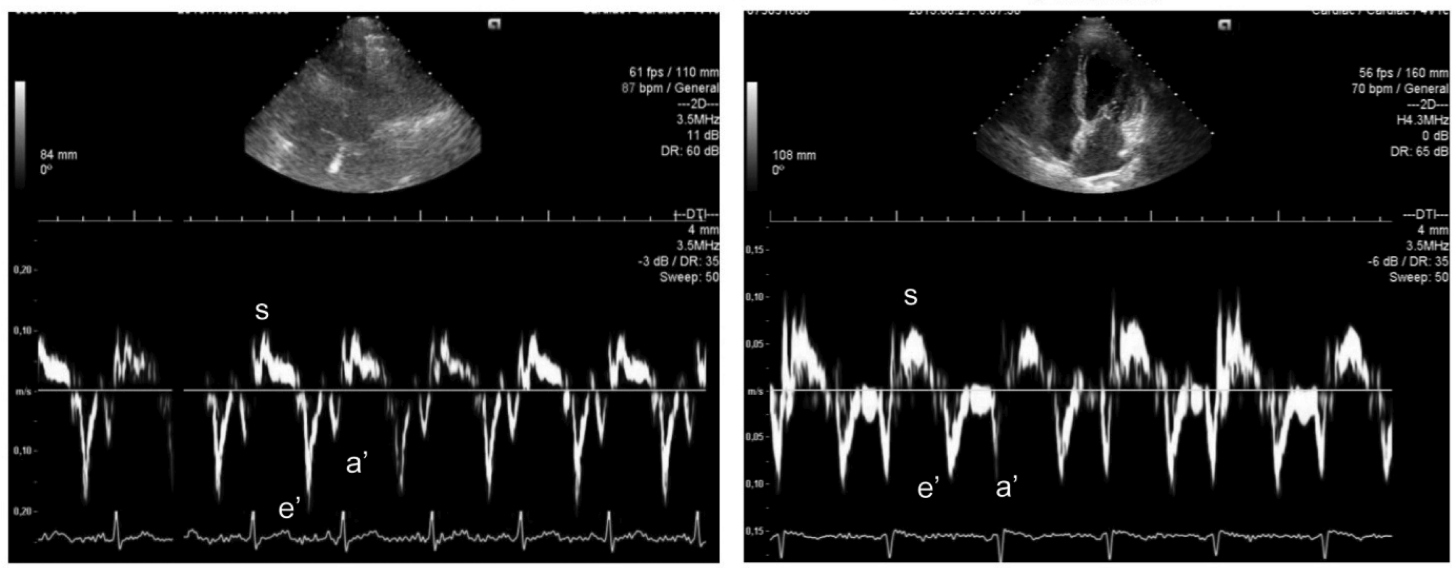

C

0 month

TAPSE

3 months
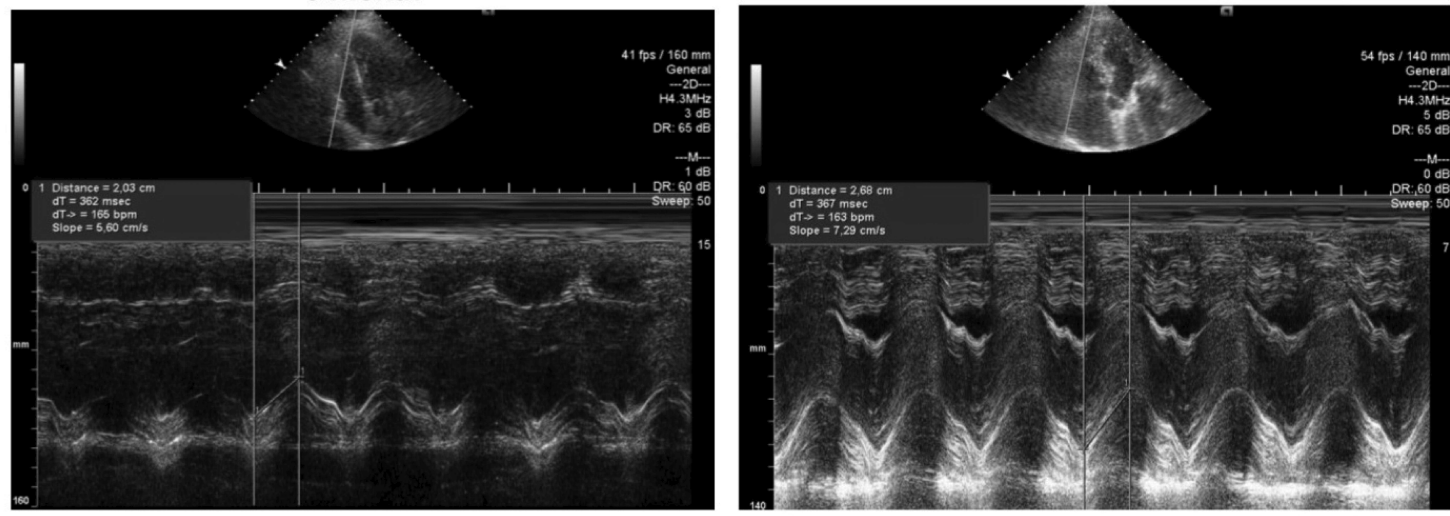

Figure 4. Representative echocardiographic images from patients with polymyositis/dermatomyositis, at the time of the diagnosis and after 3 months. Transmitral inflow (a) showed a normal pattern (E $>A$ ) at the time of diagnosis (E: peak early diastolic velocity, A: peak late diastolic velocity) but it was reversed after 3 months $(\mathrm{E}<\mathrm{A})$, suggesting diastolic dysfunction. The peak systolic velocity of the lateral annulus (lateral s) measured by tissue Doppler technique (TDI; b) revealed an initial left ventricular systolic dysfunction that normalized by the end of the followup period. The early myocardial diastolic velocity (lateral $\mathrm{e}^{\prime}$ ) decreased, while the late myocardial diastolic velocity (lateral a') increased over 3 months. The tricuspid annulus plane systolic excursion (TAPSE) was in the normal range at the time of the diagnosis and further increased during the followup (c). 
graphic diastolic variables at the end of the 3-month followup period met the requirements of the grade 1 diastolic dysfunction, i.e., impaired relaxation pattern with normal filling pressure $\left(\mathrm{A} \geq \mathrm{E}, \mathrm{E} / \mathrm{e}^{\prime} \leq 8\right.$, normal LA diameters $)^{22}$. The diastolic dysfunction that developed during steroid therapy may be the first sign of the fibrotic transformation of the myocardium. The increase in the tricuspid $\mathrm{e}^{\prime}$ and the decreasing tendency in the tricuspid $\mathrm{s}$ velocity indicated markedly different changes for the RV compared to those of the corresponding variables in the LV. This suggests a different behavior for the RV; however, we did not investigate other RV diastolic variables (e.g., tricuspid E/A, DT). More detailed studies involving large numbers of patients are needed for more precise assessment of the RV diastolic function.

The early echocardiographic diastolic abnormalities (changes in $\mathrm{E} / \mathrm{A}, \mathrm{E} / \mathrm{e}^{\prime}$ ) did not correlate with the muscle strength score, similar to previous investigators who found no correlation between echocardiographic variables and the clinical characteristics, laboratory results, or ILD ${ }^{23}$. However, Sharratt and colleagues presented a linear relationship between muscle weakness and cardiac manifestations, disease activity, and systolic time intervals on ECG in 5 patients ${ }^{24}$. A possible explanation for the conflicting data may be the difference in the patient populations and/or in the study methods. Additional prospective studies would be necessary to explore the possible relationships between the skeletal muscle and cardiac abnormalities. The exact molecular and cellular mechanisms of the LV diastolic dysfunction have not yet been clarified, but these diastolic abnormalities during the followup period suggest intrinsic myocardial manifestation ${ }^{9}$. Gupta, et al suggested the role of myocardial fibrosis and recurrent myocarditis ${ }^{25}$. Further, vascular factors such as vasculitis, intima hyperplasia, or sclerosis of the tunica media of the coronary arteries may play a role in $\mathrm{PM}^{7}$. Additionally, the specific autoantibodies (anti-Jo, anti-Ro-52, and anti-Ro-60) promote the production of interferon- $\alpha$, causing direct myofiber destruction ${ }^{26}$. Other inflammatory factors may also contribute to the development of the diastolic dysfunction. For example, interleukin 6 (IL-6), IL-1 $\beta$, and tumor necrosis factor (TNF)- $\alpha$ may cause myocyte damage through the MHC-1, by local nitric oxide release and myocardial fibrosis ${ }^{5}$.

LV diastolic dysfunction observed in the third month after the diagnosis is in agreement with previous results of other investigators $9,10,11$. Lu, et $a l^{9}$ and Wang, et $a l^{10}$ reported more severe diastolic dysfunction after 4.78 and 11.12 months of average disease duration in similar age groups. In a later study, Wang, et al found LV diastolic dysfunction frequently in DM patients without evident CV disease and reported an association between transmitral flow alteration and disease duration ${ }^{11}$. The longer disease duration seems to correlate with the persistent cardiomyocyte damage, pathologic calcification, and high concen- tration of the cytokines (e.g., vascular cell adhesion molecule, TNF- $\alpha$ ), which may all contribute to the progress of the diastolic dysfunction ${ }^{27}$.

There is increasing evidence that noninvasive cardiac imaging, including TDI and cardiac MRI, contributes to the diagnosis of myocarditis in subclinical cases and aids in the initiation of the specific treatment. Myocardial inflammation during the early stages may remain undetected by traditional echocardiography or nuclear techniques, because they cannot detect small tissue structure changes involving myocardial edema, cell infiltration, and fibrosis, which usually take place without simultaneous changes in LVEF. Cardiac MRI can detect tissue changes early in myocardial inflammation. The best imaging method is late gadolinium enhancement; it is the gold standard for the in vivo assessment of myocardial scar. It has shown excellent agreement with histology ${ }^{28}$ and is ideal for detecting small areas of necrosis missed by single photon emissioncomputed tomography or positron emission tomography ${ }^{29}$. This technique has been used to detect myocardial damage - it shows the extent and location of inflammation and fibrosis of the myocardium more clearly than do myocardial scintigraphy or echocardiography ${ }^{30}$. Cardiac MRI can predict or rule out myocardial inflammation (edema, increased membrane permeability, or capillary blood flow and fibrosis) with a diagnostic accuracy of $78 \% 31$, and fibrosis can be distinguished from ischemic necrosis ${ }^{32}$. Among echocardiography's disadvantages are operator dependency, poor acoustic window, unsuitability in cases of obesity or lung disease, and inferior reproducibility; however, compared to cardiac MRI, echocardiography is an easy, cheap, noninvasive bedside technique for ventricular function evaluation ${ }^{33}$. The appearance of novel echocardiographic modalities such as TDI, strain, myocardial perfusion imaging, and 3-D echocardiography have dramatically expanded the scope of echocardiography, providing accurate bedside assessment of regional contractility, myocardial blood flow, microvascular integrity, and longitudinal myocardial function 34,35 .

Characterization of the inflammatory process in the myocardium associated with systemic autoimmune diseases - PM/DM in our case - is of indisputable clinical importance. Only a small percentage of patients with DM/PM have clinically important cardiac disease, but heart involvement is one of the leading causes of death, and early detection remains a challenge. The initial cardiac alterations are the consequences of acute myocarditis and myocardial edema in the acute phase of the disease depicted by subclinical LV and RV dysfunction on the ECG and subepicardial late contrast enhancement on cardiac MRI. These alterations appear to be reversible in patients with specific therapy (high-dose steroid and immunosuppression), according to both our observations with echocardiography and the cardiac MRI results of Allanore, et al, who detected

Personal non-commercial use only. The Journal of Rheumatology Copyright @ 2015. All rights reserved. 
markedly reduced myocardial MRI contrast enhancement as a consequence of specific therapy ${ }^{36}$. If, however, early echocardiographic changes show progression, then recurrent inflammation, myocardial fibrosis 8,25 , and consequential diastolic dysfunction occur ${ }^{9,10,11}$. Less frequently, development of severe left or right heart systolic dysfunction indicates irreversible changes, with occurrence of severe cardiovascular pathologies (acute fulminant myocarditis, cardiomyopathy, ruptured mitral chordae tendineae, or acute right heart failure) and affecting cardiac morbidity and mortality of patients with PM/DM. Cardiac outcome in this group of patients has several determinants including age at onset of disease, sex, disease activity, time of diagnosis and initiation of specific therapy, subtype of disease, autoimmune antibody profile, associated cardiovascular and pulmonary diseases, and toxic side effects of therapy.

Our results are the initial important steps in the recognition of myocardial involvement in PM/DM. Nevertheless, our results should be interpreted in the context of certain limitations. Our study population is quite small and a larger patient number would be needed to refine our results. Identification of autoimmune myocardial involvement is difficult because of nonspecific ECG signs ${ }^{23,37}$, subclinical echocardiographic abnormalities ${ }^{38}$, and nonspecific laboratory results (elevated cTnT, CK) ${ }^{39}$. As mentioned, cardiac MRI could help diagnosis with recognition of acute myocarditis ${ }^{40}$. Endomyocardial biopsy is the gold standard in the diagnosis but being an invasive procedure, it is not routinely used. A minor limitation of our study and the TDI measurement is that tissue Doppler data are strongly affected by the angle of the ultrasound beam. To avoid errors, all variables were measured in 3 independent heart cycles and mean data were calculated.

The TDI method seems to be adequate for detection of early functional myocardial changes indicating cardiac involvement in the systemic disease. The rheumatologist, immunologist, and cardiologist should consider this, given that echocardiography with TDI is a noninvasive, safe, usually available, and relatively inexpensive technique. Echocardiographic TDI is a useful tool for the diagnosis of early cardiac abnormalities, for the prediction of the subclinical LV and RV dysfunction in the acute phase, as well as for the evaluation of LV diastolic dysfunction during disease progression.

\section{ACKNOWLEDGMENT}

We thank Judit Barta for careful reading of the manuscript.

\section{REFERENCES}

1. Fauci AS, Langford CA, Harrison TR. Harrison's rheumatology. 3rd ed. New York: McGraw-Hill Medical; 2013.

2. Oppenheim H. [On dermatomyositis.] [Article in German] Berl Klin Wochenschrift 1899;36:805-7.

3. Danko K, Ponyi A, Constantin T, Borgulya G, Szegedi G. Long-term survival of patients with idiopathic inflammatory myopathies according to clinical features: a longitudinal study of
162 cases. Medicine 2004;83:35-42.

4. Zhang L, Wang GC, Ma L, Zu N. Cardiac involvement in adult polymyositis or dermatomyositis: a systematic review. Clin Cardiol 2012;35:686-91.

5. Bazzani C, Cavazzana I, Ceribelli A, Vizzardi E, Dei Cas L, Franceschini F. Cardiological features in idiopathic inflammatory myopathies. J Cardiovasc Med 2010;11:906-11.

6. Gonzalez-Lopez L, Gamez-Nava JI, Sanchez L, Rosas E, Suarez-Almazor M, Cardona-Munoz C, et al. Cardiac manifestations in dermato-polymyositis. Clin Exp Rheumatol 1996;14:373-9.

7. Haupt HM, Hutchins GM. The heart and cardiac conduction system in polymyositis-dermatomyositis: a clinicopathologic study of 16 autopsied patients. Am J Cardiol 1982;50:998-1006.

8. Lundberg IE. The heart in dermatomyositis and polymyositis. Rheumatology 2006;45 Suppl 4:iv18-21.

9. Lu Z, Wei Q, Ning Z, Qian-Zi Z, Xiao-Ming S, Guo-Chun W. Left ventricular diastolic dysfunction - early cardiac impairment in patients with polymyositis/dermatomyositis: a tissue Doppler imaging study. J Rheumatol 2013;40:1572-7.

10. Wang H, Liu H, Yu X, Jiang H, Wang Y, Cai L. Left ventricle diastolic function in patients with polymyositis. Int J Cardiol 2013;168:4311-2.

11. Wang H, Liu HX, Wang YL, Yu XQ, Chen XX, Cai L. Left ventricular diastolic dysfunction in patients with dermatomyositis without clinically evident cardiovascular disease. J Rheumatol 2014;41:495-500.

12. Bruch C, Schmermund A, Bartel T, Schaar J, Erbel R. Tissue Doppler imaging: a new technique for assessment of pseudonormalization of the mitral inflow pattern. Echocardiography 2000;17:539-46.

13. Nagueh SF, Middleton KJ, Kopelen HA, Zoghbi WA, Quinones MA. Doppler tissue imaging: a noninvasive technique for evaluation of left ventricular relaxation and estimation of filling pressures. J Am Coll Cardiol 1997;30:1527-33.

14. Bohan A, Peter JB. Polymyositis and dermatomyositis (first of two parts). N Engl J Med 1975;292:344-7.

15. Myositis Disease Activity Assessment Tool - 2005, version 2. [Internet. Accessed October 23, 2014]; Available from: www.niehs.nih.gov/research/resources/assets/docs/myositis_disease _activity_assessment_tool_0_4_version_2_2005_pdf_format.pdf

16. Lang RM, Bierig M, Devereux RB, Flachskampf FA, Foster E, Pellikka PA, et al. Recommendations for chamber quantification: a report from the American Society of Echocardiography's Guidelines and Standards Committee and the Chamber Quantification Writing Group, developed in conjunction with the European Association of Echocardiography, a branch of the European Society of Cardiology. J Am Soc Echocardiogr 2005; 18:1440-63.

17. Armstrong WF, Ryan T, Feigenbaum H. Feigenbaum's echocardiography. 7th ed. Philadelphia: Wolters Kluwer Health/Lippincott Williams \& Wilkins; 2010

18. Yamada H, Oki T, Tabata T, Iuchi A, Ito S. Assessment of left ventricular systolic wall motion velocity with pulsed tissue Doppler imaging: comparison with peak $\mathrm{dP} / \mathrm{dt}$ of the left ventricular pressure curve. J Am Soc Echocardiogr 1998;11:442-9.

19. Oh JK, Seward JB, Tajik AJ. The echo manual. Philadelphia: Lippincott Williams \& Wilkins; 2006.

20. Ho CY, Solomon SD. A clinician's guide to tissue Doppler imaging. Circulation 2006;113:e396-8.

21. Denbow CE, Lie JT, Tancredi RG, Bunch TW. Cardiac involvement in polymyositis: a clinicopathologic study of 20 autopsied patients. Arthritis Rheum 1979;22:1088-92.

22. Nagueh SF, Appleton CP, Gillebert TC, Marino PN, Oh JK, Smiseth OA, et al. Recommendations for the evaluation of left 
ventricular diastolic function by echocardiography. J Am Soc Echocardiogr 2009;22:107-33.

23. Stern R, Godbold JH, Chess Q, Kagen LJ. ECG abnormalities in polymyositis. Arch Intern Med 1984;144:2185-9.

24. Sharratt GP, Danta G, Carson PH. Cardiac abnormality in polymyositis. Ann Rheum Dis 1977;36:575-8.

25. Gupta R, Wayangankar SA, Targoff IN, Hennebry TA. Clinical cardiac involvement in idiopathic inflammatory myopathies: a systematic review. Int J Cardiol 2011;148:261-70.

26. Eloranta ML, Barbasso Helmers S, Ulfgren AK, Ronnblom L, Alm GV, Lundberg IE. A possible mechanism for endogenous activation of the type I interferon system in myositis patients with anti-Jo-1 or anti-Ro 52/anti-Ro 60 autoantibodies. Arthritis Rheum 2007;56:3112-24.

27. Kim E, Cook-Mills J, Morgan G, Sredni ST, Pachman LM. Increased expression of vascular cell adhesion molecule 1 in muscle biopsy samples from juvenile dermatomyositis patients with short duration of untreated disease is regulated by miR-126. Arthritis Rheum 2012;64:3809-17.

28. Klein C, Nekolla SG, Bengel FM, Momose M, Sammer A, Haas F, et al. Assessment of myocardial viability with contrast-enhanced magnetic resonance imaging: comparison with positron emission tomography. Circulation 2002;105:162-7.

29. Wagner A, Mahrholdt H, Holly TA, Elliott MD, Regenfus M, Parker M, et al. Contrast-enhanced MRI and routine single photon emission computed tomography (SPECT) perfusion imaging for detection of subendocardial myocardial infarcts: an imaging study. Lancet 2003;361:374-9.

30. Aso H, Takeda K, Ito T, Shiraishi T, Matsumura K, Nakagawa T. Assessment of myocardial fibrosis in cardiomyopathic hamsters with gadolinium-DTPA enhanced magnetic resonance imaging. Invest Radiol 1998;33:22-32.

31. Gagliardi MG, Polletta B, Di Renzi P. MRI for the diagnosis and follow-up of myocarditis. Circulation 1999;99:458-9.
32. Gutberlet M, Spors B, Thoma T, Bertram H, Denecke T, Felix R, et al. Suspected chronic myocarditis at cardiac MR: diagnostic accuracy and association with immunohistologically detected inflammation and viral persistence. Radiology 2008;246:401-9.

33. Schalla S, Nagel E, Lehmkuhl H, Klein C, Bornstedt A, Schnackenburg B, et al. Comparison of magnetic resonance real-time imaging of left ventricular function with conventional magnetic resonance imaging and echocardiography. Am J Cardiol 2001;87:95-9.

34. Agati L, Tonti G, Galiuto L, Di Bello V, Funaro S, Madonna MP, et al. Quantification methods in contrast echocardiography. Eur J Echocardiogr 2005;6 Suppl 2:S14-20.

35. Leitman M, Lysyansky P, Sidenko S, Shir V, Peleg E, Binenbaum $\mathrm{M}$, et al. Two-dimensional strain-a novel software for real-time quantitative echocardiographic assessment of myocardial function. J Am Soc Echocardiogr 2004;17:1021-9.

36. Allanore Y, Vignaux O, Arnaud L, Puechal X, Pavy S, Duboc D, et al. Effects of corticosteroids and immunosuppressors on idiopathic inflammatory myopathy related myocarditis evaluated by magnetic resonance imaging. Ann Rheum Dis 2006;65:249-52.

37. Taylor AJ, Wortham DC, Burge JR, Rogan KM. The heart in polymyositis: a prospective evaluation of 26 patients. Clin Cardiol 1993;16:802-8.

38. Gottdiener JS, Sherber HS, Hawley RJ, Engel WK. Cardiac manifestations in polymyositis. Am J Cardiol 1978;41:1141-9.

39. Erlacher P, Lercher A, Falkensammer J, Nassonov EL, Samsonov MI, Shtutman VZ, et al. Cardiac troponin and beta-type myosin heavy chain concentrations in patients with polymyositis or dermatomyositis. Clin Chim Acta 2001;306:27-33.

40. Toong C, Puranik R, Adelstein S. Use of cardiac MR imaging to evaluate the presence of myocarditis in autoimmune myositis: three cases. Rheumatol Int 2012;32:779-82. 